\title{
On contraharmonic mean and Pythagorean triples
}

\author{
Jussi Pahikkala \\ Jussi Pahikkala studierte an der Universität Turku in Finnland Mathematik. Nach As- \\ sistententätigkeiten an den Universitäten von Tampere und Turku wirkte er als Gym- \\ nasiallehrer in Finnland.
}

There are many kinds of means of numbers - the most known are the arithmetic mean and the geometric mean. Already the Pythagoreans, Pythagoras and his school, knew certain means. Presumably, Pythagoras has learned in Babylonia, besides the two mentioned means, also the harmonic mean

$$
\frac{2 x y}{x+y}
$$

of the numbers $x$ and $y$. Hischer [4] calls those three means the classical Babylonian means. In addition, one may infer that the Pythagoreans had even seven other means of two numbers, as tells Hischer [4], being based on the history books [1] and [2].

By [3], three positive numbers $x, m, y$ are in contraharmonic proportion, if the ratio of the difference of the second and the first number to the difference of the third and the second number is equal the ratio of the third and the first number, i.e. if

$$
\frac{m-x}{y-m}=\frac{y}{x}
$$

Von den Pythagoräern sind uns zu zwei positiven reellen Zahlen $a, b$ mehrere Mittelbildungen überliefert. Den meisten Leserinnen und Lesern dürften das arithmetische, das geometrische sowie das harmonische Mittel wohl vertraut sein. Weniger bekannt dürfte vermutlich das sog. kontraharmonische Mittel $C(a, b)$ von $a, b$ sein, das durch die Grösse $\left(a^{2}+b^{2}\right) /(a+b)$ gegeben ist. Das kontraharmonische Mittel ist im Vergleich zu den anderen genannten Mitteln das Grösste. Der Autor geht im nachfolgenden Beitrag der Frage nach, unter welchen Bedingungen das kontraharmonische Mittel $C(a, b)$ ganzzahlig ist, sofern $a, b$ ganz sind. Im Rahmen seiner Untersuchungen findet er insbesondere einen Zusammenhang zwischen ganzzahligen kontraharmonischen Mitteln und pythagoräischen Zahlentripeln. 
The middle number $m$ is then called the contraharmonic mean (sometimes: antiharmonic mean) of the first and the last number.

The contraharmonic proportion has most likely been known in the proportion doctrine of Pythagoreans, since they have in a manner similar to (1) described the classical Babylonian means:

$$
\begin{array}{ll}
\frac{m-x}{y-m}=\frac{x}{x} & \\
\frac{m-x}{y-m}=\frac{x}{m} & (\text { arithmetic mean } m), \\
\frac{m-x}{y-m}=\frac{x}{y} & \quad(\text { heometric mean } m),
\end{array}
$$

Computing the contraharmonic mean $m$ from (1), one obtains

$$
m=\frac{x^{2}+y^{2}}{x+y}
$$

According to this model, one can define the contraharmonic mean of several positive numbers $x_{1}, \ldots, x_{n}$ :

$$
C\left(x_{1}, \ldots, x_{n}\right):=\frac{x_{1}^{2}+\ldots+x_{n}^{2}}{x_{1}+\ldots+x_{n}}
$$

\section{Comparisons}

The contraharmonic mean of two positive numbers is always between the smaller and the greater number, as is seen in the following way (suppose that $x \leq y$ ):

$$
x=\frac{x^{2}+x y}{x+y} \leq \frac{x^{2}+y^{2}}{x+y} \leq \frac{x y+y^{2}}{x+y}=y .
$$

When one compares the size of the contraharmonic and the harmonic mean of positive numbers, one sees that their difference

$$
\frac{x^{2}+y^{2}}{x+y}-\frac{2 x y}{x+y}=\frac{(x-y)^{2}}{x+y}
$$

is always nonnegative, whence the contraharmonic mean is at least equal the harmonic mean. The contraharmonic mean is a "very great" mean, since it is never below the greatest of the Babylonian means, the arithmetic mean, and it is even at least equal to the quadratic mean $\sqrt{\left(x^{2}+y^{2}\right) / 2}$. The truth of these assertions follows from the identities

$$
\frac{x^{2}+y^{2}}{x+y}-\frac{x+y}{2}=\frac{(x-y)^{2}}{2(x+y)}, \quad\left(\frac{x^{2}+y^{2}}{x+y}\right)^{2}-\left(\sqrt{\frac{x^{2}+y^{2}}{2}}\right)^{2}=\frac{\left(x^{2}+y^{2}\right)(x-y)^{2}}{2(x+y)^{2}} \text {. }
$$




\section{Integer contraharmonic means}

The contraharmonic mean

$$
c:=C(u, v)=\frac{u^{2}+v^{2}}{u+v}
$$

of two positive integers $u$ and $v$ may be an integer, too; for example, the integer 5 is the contraharmonic mean of 2 and 6 , as well as of 3 and 6, i.e. 2, 5, 6, are in contraharmonic proportion, similarly are $3,5,6$ :

$$
\frac{2^{2}+6^{2}}{2+6}=\frac{40}{8}=5=\frac{45}{9}=\frac{3^{2}+6^{2}}{3+6} .
$$

The following table gives more examples:

\begin{tabular}{||c||c|c|c|c|c|c|c|c|c|c|c|c|c|}
\hline$u$ & 2 & 3 & 3 & 4 & 4 & 5 & 5 & 6 & 6 & 6 & 6 & 7 & 7 \\
\hline$v$ & 6 & 6 & 15 & 12 & 28 & 20 & 45 & 12 & 18 & 30 & 66 & 42 & 91 \\
\hline$c$ & 5 & 5 & 13 & 10 & 25 & 17 & 41 & 10 & 15 & 26 & 61 & 37 & 85 \\
\hline
\end{tabular}

\begin{tabular}{|c|c|c|c|c|c||}
\hline 8 & 8 & 8 & 9 & 9 & $\ldots$ \\
\hline 24 & 56 & 120 & 18 & 45 & $\ldots$ \\
\hline 20 & 50 & 113 & 15 & 39 & $\ldots$ \\
\hline
\end{tabular}

The nontrivial integer contraharmonic means form Sloane's sequence A146984.

Theorem 1. For any value of $u>2$, there are at least two greater values of $v$ such that $C(u, v)$ is an integer.

Proof. The values

$$
v:=(u-1) u, \quad v:=(2 u-1) u
$$

work always because of the identities

$$
\frac{u^{2}+((u-1) u)^{2}}{u+(u-1) u}=u^{2}-2 u+2, \quad \frac{u^{2}+((2 u-1) u)^{2}}{u+(2 u-1) u}=2 u^{2}-2 u+1,
$$

the right hand sides of which are positive integers and different for $u \neq 1$. The value $u=2$ is an exception, since it has only $v=6$ with which its contraharmonic mean is an integer.

In (4), the values of $v$ are multiples of $u$, but this is not necessary to make $C(u, v)$ an integer, e.g. we have $C(10,15)=13$.

Theorem 2. If $u>1$ and $C(u, v)$ is an integer, then $u$ and $v$ have common prime divisors.

Proof. Suppose that we have positive integers $u, v$ such that $\operatorname{gcd}(u, v)=1$. Then as well, $\operatorname{gcd}(u+v, u v)=1$, since otherwise both $u+v$ and $u v$ would be divisible by a prime $p$, and thus also one of the factors $u$ and $v$ in $u v$ would be divisible by $p$; then however 
$p \mid u+v$ would imply that $p \mid u$ and $p \mid v$, whence we would have $\operatorname{gcd}(u, v) \geq p$. Consequently, we must have $\operatorname{gcd}(u+v, u v)=1$.

We make the additional assumption that $\frac{u^{2}+v^{2}}{u+v}$ is an integer, i.e. that

$$
u^{2}+v^{2}=(u+v)^{2}-2 u v
$$

is divisible by $u+v$. Therefore also $2 u v$ is divisible by this sum. But because $\operatorname{gcd}(u+$ $v, u v)=1$, the factor 2 must be divisible by $u+v$, which is at least 2 . Thus $u=v=1$.

The conclusion is, that only the "most trivial case" $u=v=1$ allows that $\operatorname{gcd}(u, v)=1$. This settles the proof.

Theorem 3. If $u$ is an odd prime number, then the values given by (4) are the only possibilities enabling integer contraharmonic means.

Proof. Let $u$ be a positive odd prime. The values $v=(u-1) u$ and $v=(2 u-1) u$ do always. As for other possible values of $v$, according to Theorem 2 , they must be multiples of the prime number $u$ :

$$
v=n u \quad(n \in \mathbb{Z})
$$

Now

$$
c=\frac{u^{2}+v^{2}}{u+v}=\frac{\left(n^{2}+1\right) u}{n+1}
$$

and since $u$ is prime, either $u \mid n+1$ or $n+1 \mid n^{2}+1$.

In the former case $n+1=k u$, one obtains

$$
c=\frac{\left(k^{2} u^{2}-2 k u+2\right) u}{k u}=k u^{2}-2 u+\frac{2}{k},
$$

which is an integer only for $k=1$ and $k=2$, corresponding to (4).

In the latter case, there must exist a prime number $p$ dividing both $n+1$ and $n^{2}+1$, whence $p \nmid n$. The equation

$$
n^{2}+1=(n+1)^{2}-2 n
$$

then implies that $p \mid 2 n$. So we must have $p \mid 2$, i.e. necessarily $p=2$. Moreover, if we had $4 \mid n+1$ and $4 \mid n^{2}+1$, then we could write $n+1=4 m$, and thus

$$
n^{2}+1=(4 m-1)^{2}+1=16 m^{2}-8 m+2 \not \equiv 0 \quad(\bmod 4),
$$

which is impossible. We infer, that now $\operatorname{gcd}\left(n+1, n^{2}+1\right)=2$, and in any case

$$
\operatorname{gcd}\left(n+1, n^{2}+1\right) \leq 2
$$

Nevertheless, since $n+1 \geq 3$ and $n+1 \mid n^{2}+1$, we should have $\operatorname{gcd}\left(n+1, n^{2}+1\right) \geqq 3$. The contradiction means that the latter case is not possible, and Theorem 3 has been proved. 
Theorem 4. If $\left(u_{1}, v, c\right)$ is a nontrivial solution of (3) with $u_{1}<c<v$, then there is always another nontrivial solution $\left(u_{2}, v, c\right)$ with $u_{2}<v$. On the other hand, if $\left(u, v_{1}, c\right)$ is a nontrivial solution with $u<c<v_{1}$, it allows no different solution $\left(u, v_{2}, c\right)$.

For example, there are the solutions $(2,6,5)$ and $(3,6,5) ;(5,20,17)$ and $(12,20,17)$.

Proof. The Diophantine equation (3) may be written

$$
u^{2}-c u+\left(v^{2}-c v\right)=0,
$$

whence

$$
u=\frac{c \pm \sqrt{c^{2}+4 c v-4 v^{2}}}{2}
$$

and the discriminant of (5) must be nonnegative because of the existence of the real root $u_{1}$. But if it were zero, i.e. if the equation $c^{2}+4 c v-4 v^{2}=0$ were true, this would imply for $v$ the irrational value $\frac{1}{2}(1+\sqrt{2}) c$. Thus the discriminant must be positive, and then also the smaller root $u$ of (5) gotten with "-" in front of the square root is positive, since we can rewrite it as

$$
\frac{c-\sqrt{c^{2}+4 c v-4 v^{2}}}{2}=\frac{c^{2}-\left(c^{2}+4 c v-4 v^{2}\right)}{2\left(c+\sqrt{c^{2}+4 c v-4 v^{2}}\right)}=\frac{2(v-c) v}{c+\sqrt{c^{2}+4 c v-4 v^{2}}},
$$

and the numerator is positive because $v>c$. Consequently, when the discriminant of the equation (5) is positive, the equation has always two distinct positive roots $u$. When one of the roots $\left(u_{1}\right)$ is an integer, the other is an integer, too, because in the numerator of (6) the sum and the difference of two integers are simultaneously even. It follows the existence of $u_{2}$, distinct from $u_{1}$.

If one solves (3) for $v$, the smaller root

$$
\frac{c-\sqrt{c^{2}+4 c u-4 u^{2}}}{2}=\frac{2(u-c) u}{c+\sqrt{c^{2}+4 c u-4 u^{2}}}
$$

is negative. Thus there cannot be any $\left(u, v_{2}, c\right)$.

One can see that all values of $c$ in the table are hypotenuses in a right triangle with integer sides. E.g., 41 is the contraharmonic mean of 5 and $45 ; 9^{2}+40^{2}=41^{2}$. So, there is an unexpected connection between the integer contraharmonic means and the Pythagorean triples:

Theorem 5. Any integer contraharmonic mean of two different positive integers is the hypotenuse of a Pythagorean triple. Conversely, any hypotenuse of a Pythagorean triple is contraharmonic mean of two different positive integers.

Proof. $1^{\circ}$. Let the integer $c$ be the contraharmonic mean

$$
c=\frac{u^{2}+v^{2}}{u+v}
$$


of the positive integers $u$ and $v$ with $u>v$. Then $u+v \mid u^{2}+v^{2}=(u+v)^{2}-2 u v$, whence

$$
u+v \mid 2 u v
$$

and we have the positive integers

$$
a:=u-v=\frac{u^{2}-v^{2}}{u+v}, \quad b:=\frac{2 u v}{u+v}
$$

satisfying

$$
\begin{aligned}
a^{2}+b^{2} & =\frac{\left(u^{2}-v^{2}\right)^{2}+(2 u v)^{2}}{(u+v)^{2}}=\frac{u^{4}-2 u^{2} v^{2}+v^{4}+4 u^{2} v^{2}}{(u+v)^{2}} \\
& =\frac{u^{4}+2 u^{2} v^{2}+v^{4}}{(u+v)^{2}}=\frac{\left(u^{2}+v^{2}\right)^{2}}{(u+v)^{2}}=c^{2} .
\end{aligned}
$$

$2^{\circ}$. Suppose that $c$ is the hypotenuse of the Pythagorean triple $(a, b, c)$, whence $c^{2}=$ $a^{2}+b^{2}$. Let us consider the rational numbers

$$
u:=\frac{c+b+a}{2}, \quad v:=\frac{c+b-a}{2} .
$$

Here one of the integers $a$ and $b$ is even but the other and $c$ are odd, or all of $a, b, c$ are even. Therefore, $c+b \pm a$ are always even, and accordingly $u$ and $v$ are positive integers. We see also that $u+v=c+b$. Now we obtain

$$
\begin{aligned}
u^{2}+v^{2} & =\frac{c^{2}+b^{2}+a^{2}+2 a b+2 b c+2 c a+c^{2}+b^{2}+a^{2}-2 a b+2 b c-2 c a}{4} \\
& =\frac{2 c^{2}+2\left(a^{2}+b^{2}\right)+4 b c}{4}=\frac{4 c^{2}+4 b c}{4}=c(c+b) \\
& =c(u+v) .
\end{aligned}
$$

Thus, $c$ is the contraharmonic mean $\frac{u^{2}+v^{2}}{u+v}$ of the different integers $u$ and $v$.

\section{References}

[1] Boyer, C.B.: A history of mathematics. Wiley \& Sons, New York 1968.

[2] Cantor, M.: Vorlesungen über die Geschichte der Mathematik. Erster Band, 2. Auflage, Teubner, Leipzig 1894.

[3] Diderot \& d'Alembert: Encyclopédie. Paris 1751-1777 (Electronic version: "L'Encyclopédie de Diderot et d'Alembert" in http: / / encyclopedie.uchicago.edu/).

[4] Hischer, H.: Viertausend Jahre Mittelwertbildung - Eine fundamentale Idee der Mathematik und didaktische Implikationen. Mathematica didactica 25 (2002), 3-51.

Jussi Pahikkala

Haunionpolku

FI-21310 Vahto, Finnland

e-mail: pahio@uusikaupunki.fi 